\title{
Soil fertility under different tillage systems in sugarcane expansion area
}

\author{
Emmerson Rodrigues de Moraes ${ }^{1}$, José Geraldo Mageste ${ }^{2}$, Joicy Vitória Miranda Peixoto ${ }^{2 *}$, Luis Augusto \\ da Silva Domingues ${ }^{3}$, Regina Maria Quintão Lana², Ernane Miranda Lemes ${ }^{2}$
}

\author{
${ }^{1}$ Instituto Federal Goiano, Campus-Morrinhos. BR 153, km 633. Zip Code: 75.650-000. Morrinhos-GO, Brazil \\ ${ }^{2}$ Universidade Federal de Uberlândia, Campus Glória. BR 050, km 78. Zip Code: 38.400-000, Uberlândia-MG, Brazil \\ ${ }^{3}$ Instituto Federal do Triângulo Mineiro, Campus Uberlândia. Fazenda Sobradinho, Postal Code: 1020. Zip Code: \\ 38400-970, Uberlândia-MG, Brazil
}

\section{*Corresponding author: joicyvmpeixoto@yahoo.com.br}

\begin{abstract}
In sugarcane expansion areas where soil fertility restrictions regularly occur, soil preparing ameliorates soil physical and chemical properties to improve conditions for sugarcane crop development. Therefore, the aim of this study was to evaluate soil chemical attributes under different soil preparation methods for sugarcane cultivation at the first and second year of expansion area in the Cerrado biome. The experiment was conducted in an area previously used as pasture land for more than 10 years with Brachiaria decumbens without any soil correction. СTC-2 sugarcane variety was planted. The experiment was set up as a randomized block design with six treatments and four replications. The treatments were: desiccation-liming-plowing-harrowing; liming-plowingharrowing; liming-harrowing-plowing-harrowing; desiccation-liming-direct planting; desiccation-liming-subsoiling, and harrowingliming-plowing-harrowing. Soil attributes: organic matter, water $\mathrm{pH}, \mathrm{H}^{+}+\mathrm{Al}^{3+}, \mathrm{Al}^{3+}, \mathrm{m}, \mathrm{V}, \mathrm{H}_{2} \mathrm{PO}_{4}^{-}, \mathrm{K}^{+}, \mathrm{Ca}^{2+}, \mathrm{Mg}^{2+}$ and $\mathrm{S}-\mathrm{SO}_{4}^{-2}$ were evaluated at 0-0.2, 0.2-0.4 and 0.4-0.6 m soil depth. The variables were submitted ANOVA, joint analysis and Tukey's test $(p<0.05)$. The treatments including liming followed by harrowing, plowing and harrowing, and harrowing followed by liming, plowing and harrowing, resulted in the largest gains in soil fertility. In the first year of sugarcane cultivation, the no-tillage system proved to be sustainable and appropriate for sugarcane cultivation economically viable.
\end{abstract}

Keywords: Brazilian savanna; no-till farming; Saccharum spp.; soil management; soil nutrient availability.

\section{Introduction}

The sugarcane crop cultivation is a widespread activity in Brazil, with the greatest productions coming from São Paulo, Goiás, Minas Gerais, Mato Grosso do Sul, Paraná, Pernambuco and Alagoas states. The Brazilian average yield is estimated in about $72.17 \mathrm{t} \mathrm{ha}^{-1}$ for the 2014/15 crop season, which was $2.37 \%$ higher than previous crop season; the total production in 2016 was approximately 657.2 million tons (CONAB, 2017), making sugarcane the most productive crop in Brazil. Sugarcane plantation is expanding mainly into areas previously used as pasture for cattle rising where no fertilization or soil correction are regularly applied. According to Inácio and Santos (2014), the cultivation of sugarcane is overlapping agricultural activities such as livestock, coffee, and soybeans areas.

In these areas, questions raised about which soil preparation should be adopted to improve the productivity and sustainability of the sugarcane plantation. The conventional tillage and the conservationist soil management called notillage, or no-till, require adaptations for sugarcane crop. As this culture is semi-permanent, rotating crops expected to happen in a no-tillage system of soil management does not occur, therefore sugarcane no-tillage is called minimum cultivation system, which does not always bring benefits to the sugarcane production system (Cury et al. 2014).
Benedini and Conde (2008) mention that the excessive soil disturbance during traditional preparation affects rainwater flow, hindering infiltration and increasing runoff, leading to soil erosion. On the other hand, production systems that prioritize soil stability as the no-tillage, tend to predominate in these areas, but with adaptations such as the removal of terraces for mechanical harvesting, although not recommended.

Foltran (2008), studying the application of lime, calcium silicate and gypsum in sugarcane ratoon without burning, showed that lime application without its incorporation in notill system achieved the most promising results. However, researches describing the best conditions for the application of lime in sugarcane are scarce in the scientific literature. Soil preparation aims to minimize adverse conditions and to obtain maximum productivity by providing an adequate environment for root development. Physical, chemical, biological and soil external factors such as infestations by weeds must be considered when defining the best strategy for soil management. Areas without physical, chemical or biological limitations provide great sugarcane yields and profits when no-tillage system was adopted (Vitti and Mazza, 2002). 
Thus, this study aimed to investigate variations in soil chemical properties, organic matter, and macronutrients in areas cultivated with different soil revolving managements before sugarcane planting in an expansion cropping area at the Brazilian Savanna biome (Cerrado).

\section{Results and discussion}

\section{Soil acidity}

No significant differences $(p>0.05)$ were observed for soil water $\mathrm{pH}$ among soil preparing treatments evaluated in the year 2010 (Table 2). However, the average soil pH at the 0$0.2 \mathrm{~m}$ soil layer was 5.1 , which was classified as "low soil pH" with high active acidity, according to Alvarez et al. (1999). The harrowing before liming and subsequent plowing and harrowing again (T6) presented a soil pH of 5.5 at the same soil layer, which is considered as "good soil pH" (Alvarez V. et al., 1999). The T6 treatment tended to increase soil $\mathrm{pH}$ value improving its agronomic soil class of interpretation, compared to the initial soil $\mathrm{pH}$ condition.

The soil water $\mathrm{pH}$ observed in 2011 at $0-0.2 \mathrm{~m}$ soil depth was low for treatments 'liming-plowing-harrowing' (T2) and 'desiccation-liming-direct planting' (T4). Since T2 treatment was not desiccated, there was great $\mathrm{Ca}^{2+}$ and $\mathrm{Mg}^{2+}$ absorption by weeds and by ratoon sugarcane consequently reducing the levels of these bases in the soil solution. In T4, the low soil water $\mathrm{pH}$ can be due to the no-tillage soil management, which did not incorporate the lime and reduce its reaction with the soil components. Freitas et al. (2017) evaluated the soil chemical properties from conventional sugarcane crop area and from native area and reported that the highest and lowest soil $\mathrm{pH}$ occurred in areas with soil revolving and in soils from undisturbed areas, respectively. The soil treatments did not affect the soil water $\mathrm{pH}$ of the 0.2-0.4 m soil depth in both years and at 0.4-0.6 $\mathrm{m}$ soil depth in the year 2010 (Table 2). The soil pH at 0.4-0.6 m soil depth in the year 2011 was great at the T6 treatment. This can be explained by the better incorporation of lime in the soil at greater depths due to great soil revolving (harrowing-limingplowing-harrowing). The application of lime plus combinations of plowing and harrowing can influence soil water $\mathrm{pH}$ at $0-0.2 \mathrm{~m}$ soil depth (Table 2). Also, in this superficial soil layer is where most of the root system is located and consequently where there is the greatest base extraction from the soil solution. In great soil depths (>0.2 $\mathrm{m})$ the soil bases are low and therefore the acidity is great (low soil pH) (Raij, 2011).

The levels of $\mathrm{H}^{+}+\mathrm{Al}^{3+}$ were great at 0-0.2 $\mathrm{m}$ soil depth of the T2 treatment in both years (Table 2). This can be explained by the great presence of $\mathrm{Al}^{3+}\left(2.06 \mathrm{cmolc} \mathrm{dm}^{-3}\right.$ in 2010 and

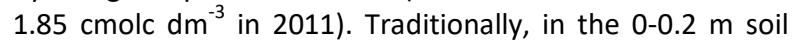
depth, there was better lime incorporation, but it did not fully reach 0.2-0.4 and 0.4-0.6 m soil layers. Reductions in the potential acidity are usually observed at 0-0.2 and 0.2$0.4 \mathrm{~m}$ soil depth and may be directly attributed to the benefits of lime, which reacts soil carbonate anions $\left(\mathrm{CO}_{3}^{-2}\right.$ and $\mathrm{HCO}_{3}{ }^{-}$) with soil potential acidity $\left(\mathrm{H}^{+}+\mathrm{Al}^{3+}\right)$ (Okorkov and Okorkova, 2013).

At 0.2-0.4 $\mathrm{m}$ soil depth the levels of $\mathrm{H}^{+}+\mathrm{Al}^{3+}$ were great for T4 (desiccation-liming-direct planting) in 2010, and for T3 (liming-harrowing-plowing-harrowing) in both years (Table 2). This can be argued by the great soil water $\mathrm{pH}$ where $\mathrm{T3}$ treatment was applied, being 5.05 in 2010 and 4.62 in 2011; in $\mathrm{T} 4$, a great concetration of $\mathrm{Al}^{3+}\left(2.03 \mathrm{cmolc}^{-3} \mathrm{dm}^{-3}\right)$ was observed in the first year. As for 0.4-0.6 $\mathrm{m}$ soil depth, no influence on the levels of $\mathrm{H}^{+}+\mathrm{Al}^{3+}$ was observed among treatments.

When working with soil revolving it is expected that implements like moldboard plows reach soil depths of up to $0.3 \mathrm{~m}$, in the range of most root system, incorporating lime and improving its reaction in soil (Kaminski et al., 2007). Weirich Neto et al. (2000) found a significant effect when lime was incorporated up to $0.15 \mathrm{~m}$ deep. Duiker and Beegle (2006) using moldboard plows incorporate lime at 0.3-0.35 $\mathrm{m}$ soil depth and demonstrated that this strategy significantly increases $\mathrm{Ca}^{2+}$ availability and reduces the $\mathrm{Al}^{3+}$ toxicity improving soil characteristics for root development in deep soil layers.

The levels of $\mathrm{H}^{+}+\mathrm{Al}^{3+}$ at 0-0.2 $\mathrm{m}$ soil depth were low for T6 (harrowing-liming-plowing-harrowing) treatment (Table 2). An explanation for this significant reduction is when first harrowing is done, before liming, the revolved soil exposed great contact surface area, therefore, with subsequent plowing and harrowing, the lime incorporation was efficiently done, and thus, the levels of $\mathrm{H}^{+}+\mathrm{Al}^{3+}$ were reduced. Weirich Neto et al. (2000) observed increased maize productivity when lime was half dose distributed on the soil surface, then incorporated by plowing, followed by the application of the other half dose, with two subsequent harrowing labors.

At the 0.2-0.4 $\mathrm{m}$ soil depth, it was observed similar results with an increase of $\mathrm{H}^{+}+\mathrm{Al}^{3+}$ in almost all treatments, with the exception of $\mathrm{T} 2$, in both evaluated years. While in the 0.4-0.6 m soil depth great $\mathrm{H}^{+}+\mathrm{Al}^{3+}$ level was superior for T4, $\mathrm{T} 5$ and $\mathrm{T} 6$ treatments, which is correlated with the low $\mathrm{pH}$ found at this soil depth.

The levels of $\mathrm{Ca}^{2+}$ and the $\mathrm{Al}^{3+}$ saturation were not influenced from one year to another (Table 2). The $\mathrm{Al}^{3+}$ and base saturation have a direct correlation with the $\mathrm{Al}^{3+}$ and cation content in the soil profile (Novais, 2007). The $\mathrm{Al}^{3+}$ level at 0-0.2 $\mathrm{m}$ soil depth was low in 2010 for the treatments T5 and T6 (Table 2). While in 2011, the $\mathrm{Al}^{3+}$ levels were low for the treatments $\mathrm{T} 3$ and T6. These results indicate that T6 treatment is more efficient in lime incorporation since the soil was heavily revolved (harrowingliming-plowing-harrowing). This incorporation is a regular practice in agriculture to raise soil $\mathrm{pH}$ at deep soil layers, to increase soil exchangeable cations and quick complex exchangeable soil acidity (Rossielo and Jacob Neto, 2006).

At $0.2-0.4 \mathrm{~m}$ soil depth the T3 treatment presented low levels of $\mathrm{Ca}^{2+}$ in both years (Table 2). This result can be due to the low $\mathrm{H}^{+}+\mathrm{Al}^{3+}$ levels and great soil water $\mathrm{pH}$. At the 0.4$0.6 \mathrm{~m}$ soil layer there was no influence of the treatments in the $\mathrm{Al}^{3+}$ level in 2010, while the $\mathrm{T} 1$ treatment was effective in reducing $\mathrm{Al}^{3+}$ level in 2011. This result is explained by the lime incorporation, improving lime reaction with soil, and the weed desiccation, which eliminates plants that could absorb soil bases and decrease soil $\mathrm{pH}$, increasing $\mathrm{Al}^{3+}$ levels. Differences in $\mathrm{Al}^{3+}$ saturation in the 0.2-0.4 and 0.4-0.6 $\mathrm{m}$ soil 
Table 1. Soil physical characteristics of the experimental area at 0-0.2 and 0.2-0.4 $\mathrm{m}$ soil depth.

\begin{tabular}{|c|c|c|c|c|c|}
\hline Depth (m) & CS & $\begin{array}{c}\mathrm{FS} \\
\mathrm{kg}^{-1}-\end{array}$ & Silt & Clay & Texture Class $^{1}$ \\
\hline $0-0.2$ & 77 & 284 & 159 & 480 & Clay soil \\
\hline $0.2-0.4$ & 122 & 206 & 139 & 533 & Clay soil \\
\hline
\end{tabular}

CS: coarse sand; FS: fine sand. 1: pipette method (EMBRAPA, 2017).

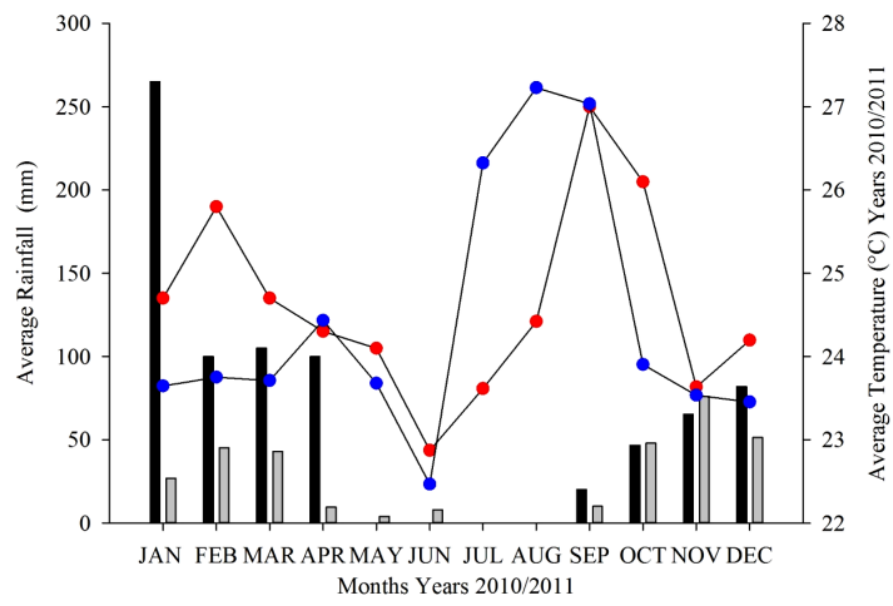

$$
\begin{aligned}
& \text { Average Rainfall }(\mathrm{mm}) \text { of the Year } 2010 \\
& \text { Average Rainfall }(\mathrm{mm}) \text { of the Year } 2011 \\
& - \text { Months vs Average Temperature }\left({ }^{\circ} \mathrm{C}\right) \text { of the year } 2010 \\
& - \text { Months vs Average Temperature }\left({ }^{\circ} \mathrm{C}\right) \text { of the year } 2011
\end{aligned}
$$

\begin{tabular}{|c|c|c|c|c|c|c|c|c|c|}
\hline Depth (m) & Treatment & \multicolumn{2}{|l|}{$\mathrm{pH}\left(\mathrm{H}_{2} \mathrm{O}\right)$} & \multicolumn{2}{|l|}{$\mathrm{H}^{+}+\mathrm{Al}^{3+}$} & \multicolumn{2}{|c|}{$\mathrm{Al}^{3+}$} & \multicolumn{2}{|c|}{ m } \\
\hline \multirow{2}{*}{\multicolumn{2}{|c|}{ Initial Condition $^{1}$ (Year 2009) }} & 4.01 & & & & & & & \\
\hline & & 2010 & 2011 & 2010 & 2011 & 2010 & 2011 & 2010 & 2011 \\
\hline \multirow{6}{*}{$0-0.2$} & T1 & $5.10 \mathrm{aA}^{2}$ & $95 \mathrm{aA}$ & $4.97 \mathrm{aA}$ & $5.47 \mathrm{aA}$ & $1.60 \mathrm{bA}$ & $1.45 \mathrm{bA}$ & $60.59 \mathrm{bA}$ & $47.15 \mathrm{bA}$ \\
\hline & $\mathrm{T} 2$ & $4.95 \mathrm{aA}$ & $4.70 \mathrm{bA}$ & $5.87 \mathrm{bA}$ & $6.97 \mathrm{bA}$ & $2.06 \mathrm{cA}$ & $1.85 \mathrm{cA}$ & $75.62 \mathrm{bA}$ & $64.88 \mathrm{cA}$ \\
\hline & T3 & $5.10 \mathrm{aA}$ & $5.07 \mathrm{aA}$ & $4.22 \mathrm{aA}$ & $4.35 \mathrm{aA}$ & $1.12 \mathrm{bA}$ & $0.97 \mathrm{aA}$ & $37.49 \mathrm{aA}$ & $24.71 \mathrm{aA}$ \\
\hline & T4 & $4.92 \mathrm{aA}$ & $4.65 \mathrm{bA}$ & $4.20 \mathrm{aA}$ & $5.40 \mathrm{aA}$ & $1.31 \mathrm{bA}$ & $1.32 \mathrm{bA}$ & $45.81 \mathrm{aA}$ & $44.00 \mathrm{bA}$ \\
\hline & T5 & $5.02 \mathrm{aA}$ & $4.97 \mathrm{aA}$ & $4.52 \mathrm{aA}$ & $5.67 \mathrm{aA}$ & $0.97 \mathrm{aA}$ & $1.22 \mathrm{bA}$ & $34.58 \mathrm{aA}$ & $36.83 \mathrm{bA}$ \\
\hline & T6 & $5.50 \mathrm{aA}$ & $5.02 \mathrm{aB}$ & $3.45 \mathrm{aB}$ & $5.05 \mathrm{aA}$ & $0.48 \mathrm{aA}$ & $0.95 \mathrm{aA}$ & $16.46 \mathrm{aA}$ & $27.37 \mathrm{aA}$ \\
\hline \multirow{2}{*}{\multicolumn{2}{|c|}{ Initial Condition ${ }^{1}$ (Year 2009) }} & 3.97 & & 8.70 & & 2.00 & & 82 & \\
\hline & & 2010 & 2011 & 2010 & 2011 & 2010 & 2011 & 2010 & 2011 \\
\hline \multirow{6}{*}{$0.2-0.4$} & T1 & $4.77 \mathrm{aA}$ & $4.55 \mathrm{bA}$ & $5.45 \mathrm{bB}$ & $6.72 \mathrm{bA}$ & $2.20 \mathrm{cA}$ & $1.85 \mathrm{cA}$ & $77.39 \mathrm{bA}$ & $68.36 \mathrm{cA}$ \\
\hline & $\mathrm{T} 2$ & $4.82 \mathrm{aA}$ & $4.57 \mathrm{bA}$ & $5.87 \mathrm{bA}$ & 7.02 bA & $2.27 \mathrm{cA}$ & $1.95 \mathrm{cA}$ & $83.10 \mathrm{bA}$ & $78.08 \mathrm{cA}$ \\
\hline & T3 & $5.05 \mathrm{aA}$ & $4.62 \mathrm{bB}$ & $4.50 \mathrm{aB}$ & $6.05 \mathrm{aA}$ & $1.41 \mathrm{bA}$ & $1.40 \mathrm{bA}$ & $47.10 \mathrm{aA}$ & $41.41 \mathrm{bA}$ \\
\hline & T4 & $4.95 \mathrm{aA}$ & $4.55 \mathrm{bB}$ & $4.90 \mathrm{aB}$ & $6.70 \mathrm{bA}$ & $2.23 \mathrm{cA}$ & $2.02 \mathrm{cA}$ & 86.17 bA & $79.44 \mathrm{cA}$ \\
\hline & T5 & $4.85 \mathrm{aA}$ & $4.47 \mathrm{bB}$ & $5.90 \mathrm{bB}$ & 7.55 bA & $2.25 \mathrm{cA}$ & $2.00 \mathrm{cA}$ & $78.97 \mathrm{bA}$ & $68.78 \mathrm{cA}$ \\
\hline & T6 & $4.80 \mathrm{aA}$ & $4.52 \mathrm{bA}$ & $5.70 \mathrm{bB}$ & $7.10 \mathrm{bA}$ & $2.22 \mathrm{cA}$ & $1.95 \mathrm{cA}$ & $71.69 \mathrm{bA}$ & $62.95 \mathrm{cA}$ \\
\hline \multirow{6}{*}{$0.4-0.6$} & T1 & $4.67 \mathrm{aA}$ & $4.57 \mathrm{bA}$ & $4.70 \mathrm{aA}$ & $5.40 \mathrm{aA}$ & $1.96 \mathrm{cA}$ & $1.52 \mathrm{bA}$ & $80.85 \mathrm{bA}$ & $69.63 \mathrm{cA}$ \\
\hline & $\mathrm{T} 2$ & $4.95 \mathrm{aA}$ & $4.67 \mathrm{bA}$ & $5.07 \mathrm{aA}$ & $5.82 \mathrm{aA}$ & $2.07 \mathrm{cA}$ & $1.75 \mathrm{cA}$ & $85.19 \mathrm{bA}$ & $80.48 \mathrm{cA}$ \\
\hline & T3 & $4.95 \mathrm{aA}$ & $4.72 \mathrm{bA}$ & $4.57 \mathrm{aA}$ & $5.80 \mathrm{aA}$ & $1.80 \mathrm{cA}$ & $1.65 \mathrm{cA}$ & $71.02 \mathrm{bA}$ & $65.27 \mathrm{cA}$ \\
\hline & T4 & $4.97 \mathrm{aA}$ & $4.62 \mathrm{bB}$ & $4.37 \mathrm{aB}$ & $5.77 \mathrm{aA}$ & $2.10 \mathrm{cA}$ & $1.85 \mathrm{cA}$ & $91.19 \mathrm{bA}$ & $83.37 \mathrm{cA}$ \\
\hline & T5 & $4.97 \mathrm{aA}$ & $4.65 \mathrm{bB}$ & $4.92 \mathrm{aB}$ & $6.25 \mathrm{aA}$ & $2.10 \mathrm{cA}$ & $1.75 \mathrm{cA}$ & $82.15 \mathrm{bA}$ & $72.58 \mathrm{cA}$ \\
\hline & T6 & $5.07 \mathrm{aA}$ & $4.80 \mathrm{aA}$ & $4.90 \mathrm{aB}$ & $6.17 \mathrm{aA}$ & $2.06 \mathrm{cA}$ & $1.87 \mathrm{cA}$ & $87.12 \mathrm{bA}$ & $79.19 \mathrm{cA}$ \\
\hline
\end{tabular}

Fig 1. Average rainfall $(\mathrm{mm})$ and temperature $\left({ }^{\circ} \mathrm{C}\right)$ at the experimental site. Source: Jalles Machado Mill (2010 and 2011).

Table 2. Soil water $\mathrm{pH}$, potential acidity $\left(\mathrm{H}^{+}+\mathrm{Al} \mathrm{I}^{3+}\right)$, exchangeable $\left(\mathrm{Al} \mathrm{I}^{3+}\right)$ and $\mathrm{Al^{3+ }}$ saturation $(\mathrm{m})$ for different tillage systems.

${ }^{1}:$ Soil initial conditions before the experiment establishment. Data only for 0-0.2 $\mathrm{m}$ and $0.2-0.4 \mathrm{~m}$ soil depth

2: Means followed by different lowercase letters in the column and means followed by different capital letters in the line differ statistically by F and Scott-Knott test at $5 \%$ probability. Treatment: $T 1$ : desiccation-liming-plowing-harrowing-planting; T2: liming-plowing-harrowing; T3: liming-harrowing-plowing-harrowing; T4: desiccation-liming-direct planting; T5: desiccation-liming-subsoiling; T6: harrowing-liming-plowing-harrowing. na: not available. 
Table 3. Exchangeable cations and soil base saturation (V) for different tillage systems.

\begin{tabular}{|c|c|c|c|c|c|c|c|c|c|}
\hline \multirow{2}{*}{$\begin{array}{l}\text { Depth }(\mathrm{m}) \\
\text { Initial Cond }\end{array}$} & \multirow{2}{*}{$\begin{array}{l}\text { Treatment } \\
1^{1} \text { (Year 2009) }\end{array}$} & \multicolumn{2}{|l|}{$\mathrm{Ca}^{2+}$} & $\mathrm{Mg}^{2+}$ & & \multicolumn{2}{|c|}{$\mathrm{K}^{+}$} & \multicolumn{2}{|c|}{$\begin{array}{l}\mathrm{V} \\
-\end{array}$} \\
\hline & & \multicolumn{4}{|l|}{0.45} & \multicolumn{2}{|c|}{78.00} & \multicolumn{2}{|c|}{10.25} \\
\hline & & 2010 & 2011 & 2010 & 2011 & 2010 & 2011 & 2010 & 2011 \\
\hline \multirow{6}{*}{$0-0.2$} & $\mathrm{~T} 1$ & $0.70 \mathrm{bA}^{2}$ & $1.10 \mathrm{bA}$ & $0.27 \mathrm{cB}$ & $0.57 \mathrm{bA}$ & $62.00 \mathrm{aA}$ & $0.50 \mathrm{aA}$ & $17,68 \mathrm{cA}$ & $26.00 \mathrm{bA}$ \\
\hline & $\mathrm{T} 2$ & $0.25 \mathrm{aA}$ & $0.50 \mathrm{cA}$ & $0.22 \mathrm{cA}$ & $0.32 \mathrm{cA}$ & $73.25 \mathrm{aA}$ & $71.75 \mathrm{aA}$ & $10,22 \mathrm{cA}$ & $12.55 \mathrm{cA}$ \\
\hline & $\mathrm{T} 3$ & $1.12 \mathrm{aA}$ & $1.87 \mathrm{aA}$ & $0.57 \mathrm{bB}$ & $0.92 \mathrm{aA}$ & $68.50 \mathrm{aA}$ & $67.00 \mathrm{aA}$ & $31.35 \mathrm{bA}$ & $40.49 \mathrm{aA}$ \\
\hline & $\mathrm{T} 4$ & $1,00 \mathrm{aA}$ & $1.02 \mathrm{bA}$ & $0.50 \mathrm{bA}$ & $0.55 \mathrm{bA}$ & $46.75 \mathrm{bA}$ & $45.25 \mathrm{bA}$ & $27.25 \mathrm{bA}$ & $23.85 \mathrm{bA}$ \\
\hline & T5 & $1.27 \mathrm{aA}$ & $1.40 \mathrm{bA}$ & $0.55 \mathrm{bA}$ & $0.72 \mathrm{bA}$ & $45.00 \mathrm{bA}$ & $58.75 \mathrm{aA}$ & $28.38 \mathrm{bA}$ & $28.52 \mathrm{bA}$ \\
\hline & T6 & $1.72 \mathrm{aA}$ & $1.65 \mathrm{aA}$ & $0.92 \mathrm{aA}$ & $0.90 \mathrm{aA}$ & 57.75 aA & $68.50 \mathrm{aA}$ & $44.23 \mathrm{aA}$ & $35.63 \mathrm{aA}$ \\
\hline \multirow{2}{*}{\multicolumn{2}{|c|}{ Initial Condition $^{1}$ (Year 2009) }} & 0.23 & & 0.15 & & 19.20 & & 4.80 & \\
\hline & & 2010 & 2011 & 2010 & 2011 & 2010 & 2011 & 2010 & 2011 \\
\hline \multirow{6}{*}{$0.2-0.4$} & $\mathrm{~T} 1$ & $0.30 \mathrm{bA}$ & $0.57 \mathrm{cA}$ & $0.25 \mathrm{cA}$ & $0.25 \mathrm{cA}$ & $36.50 \mathrm{bA}$ & $44.50 \mathrm{bA}$ & $10.29 \mathrm{cA}$ & $12.16 \mathrm{cA}$ \\
\hline & $\mathrm{T} 2$ & $0.20 \mathrm{bA}$ & $0.27 \mathrm{cA}$ & $0.15 \mathrm{cA}$ & $0.17 \mathrm{cA}$ & $37.75 \mathrm{bA}$ & $41.00 \mathrm{bA}$ & $7.41 \mathrm{cA}$ & $7.20 \mathrm{cA}$ \\
\hline & $\mathrm{T} 3$ & $1.15 \mathrm{aA}$ & $1.35 \mathrm{bA}$ & $0.52 \mathrm{bA}$ & $0.60 \mathrm{bA}$ & $35.25 \mathrm{bA}$ & $47.50 \mathrm{bA}$ & $28.48 \mathrm{bA}$ & $25.90 \mathrm{bA}$ \\
\hline & $\mathrm{T} 4$ & $0.17 \mathrm{bA}$ & $0.30 \mathrm{cA}$ & $0.12 \mathrm{cA}$ & $0.15 \mathrm{cA}$ & $24.50 \mathrm{bA}$ & $30.25 \mathrm{cA}$ & $6.81 \mathrm{cA}$ & $7.31 \mathrm{cA}$ \\
\hline & T5 & $0.32 \mathrm{bA}$ & $0.55 \mathrm{cA}$ & $0.17 \mathrm{cA}$ & $0.25 \mathrm{cA}$ & $34.00 \mathrm{bA}$ & $43.75 \mathrm{bA}$ & $9.19 \mathrm{cA}$ & $10.85 \mathrm{cA}$ \\
\hline & T6 & $0.50 \mathrm{bA}$ & $0.70 \mathrm{cA}$ & $0.30 \mathrm{cA}$ & $0.35 \mathrm{cA}$ & $33.00 \mathrm{bA}$ & $39.50 \mathrm{bA}$ & $13.42 \mathrm{cA}$ & $13.92 \mathrm{cA}$ \\
\hline \multirow{6}{*}{$0.4-0.6$} & $\mathrm{~T} 1$ & $0.27 \mathrm{bA}$ & $0.47 \mathrm{cA}$ & $0.12 \mathrm{cA}$ & $0.17 \mathrm{cA}$ & $28.50 \mathrm{bA}$ & $30.50 \mathrm{cA}$ & $8.86 \mathrm{cA}$ & $11.41 \mathrm{cA}$ \\
\hline & $\mathrm{T} 2$ & $0.15 \mathrm{bA}$ & $0.20 \mathrm{cA}$ & $0.12 \mathrm{cA}$ & $0.15 \mathrm{cA}$ & $34.75 \mathrm{bA}$ & $31.25 \mathrm{cA}$ & $6.80 \mathrm{cA}$ & $6.77 \mathrm{cA}$ \\
\hline & $\mathrm{T} 3$ & $0.50 \mathrm{bA}$ & $0.62 \mathrm{cA}$ & $0.20 \mathrm{cA}$ & $0.25 \mathrm{cA}$ & $31.75 \mathrm{bA}$ & $35.75 \mathrm{cA}$ & $14.71 \mathrm{cA}$ & $14.15 \mathrm{cA}$ \\
\hline & $\mathrm{T} 4$ & $0.12 \mathrm{bA}$ & $0.20 \mathrm{cA}$ & $0.02 \mathrm{cA}$ & $0.10 \mathrm{cA}$ & $22.00 \mathrm{bA}$ & $26.50 \mathrm{cA}$ & $4.40 \mathrm{cA}$ & $6.00 \mathrm{cA}$ \\
\hline & T5 & $0.27 \mathrm{bA}$ & $0.45 \mathrm{cA}$ & $0.12 \mathrm{cA}$ & $0.17 \mathrm{cA}$ & $25.00 \mathrm{bA}$ & $34.25 \mathrm{cA}$ & $8.68 \mathrm{cA}$ & $9.85 \mathrm{cA}$ \\
\hline & T6 & $0.15 \mathrm{bA}$ & $0.27 \mathrm{cA}$ & $0.10 \mathrm{cA}$ & $0.15 \mathrm{cA}$ & $24.50 \mathrm{bA}$ & $29.50 \mathrm{cA}$ & $5.77 \mathrm{cA}$ & $7.37 \mathrm{cA}$ \\
\hline
\end{tabular}

2. Means followed by different lowercase letters in the column and means followed by different capital letters in the line differ statistically by $F$ and Scott-Knott test at $5 \%$ probability. Treatment: $T 1$ : desiccation-liming-plowing-harrowing-planting; T2: liming-plowing-harrowing; T3: liming-harrowing-plowing-harrowing; T4: desiccation-liming-direct planting; T5: desiccation-liming-subsoiling; T6: harrowing-liming-plowing-harrowing.

Table 4. Soil phosphorus, sulfur, and organic matter (OM) for different tillage systems.

\begin{tabular}{|c|c|c|c|c|c|c|c|}
\hline \multirow{3}{*}{\multicolumn{2}{|c|}{$\begin{array}{l}\text { Depth (m) Treatment } \\
\text { Initial Condition }^{1} \text { (Year 2009) }\end{array}$}} & \multirow{2}{*}{$\begin{array}{l}\mathrm{H}_{2} \mathrm{PO}_{4} \\
-1.40\end{array}$} & $g \mathrm{dm}^{-3}$ & \multirow{2}{*}{$\begin{array}{l}\mathrm{S}^{-\mathrm{SO}_{4}}{ }^{-2} \\
4\end{array}$} & \multirow{2}{*}{\multicolumn{3}{|c|}{ 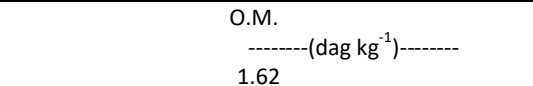 }} \\
\hline & & & & & & & \\
\hline & & 2010 & 2011 & 2010 & 2011 & 2010 & 2011 \\
\hline \multirow{6}{*}{$0-0.2$} & $\mathrm{~T} 1$ & $1.02 \mathrm{bA}^{2}$ & $1.00 \mathrm{bA}$ & $18.25 \mathrm{bA}$ & $18.00 \mathrm{cA}$ & $1.55 \mathrm{aA}$ & $1.82 \mathrm{aA}$ \\
\hline & $\mathrm{T} 2$ & $1.10 \mathrm{bA}$ & $0.75 \mathrm{bA}$ & $15.75 \mathrm{bA}$ & $8.50 \mathrm{dA}$ & $1.52 \mathrm{aA}$ & $1.85 \mathrm{aA}$ \\
\hline & T3 & $3.50 \mathrm{aA}$ & $2.53 \mathrm{aA}$ & $3.25 \mathrm{bB}$ & $20.37 c A$ & $1.67 \mathrm{aA}$ & $2.00 \mathrm{aA}$ \\
\hline & T4 & $4.70 \mathrm{aA}$ & $1.98 \mathrm{aB}$ & $13.00 \mathrm{bB}$ & $31.50 \mathrm{bA}$ & $1.55 \mathrm{aA}$ & $1.57 \mathrm{bA}$ \\
\hline & T5 & $3.47 \mathrm{aA}$ & $1.53 \mathrm{bA}$ & $16.50 \mathrm{bA}$ & $29.00 \mathrm{bA}$ & $1.80 \mathrm{aA}$ & $2.02 \mathrm{aA}$ \\
\hline & T6 & $1.16 \mathrm{bA}$ & $2.44 \mathrm{aA}$ & $36.50 \mathrm{aA}$ & $32.00 \mathrm{bA}$ & $1.80 \mathrm{aA}$ & $1.97 \mathrm{aA}$ \\
\hline \multirow{2}{*}{\multicolumn{2}{|c|}{ Initial Condition $^{1}$ (Year 2009) }} & 0.70 & & 16.00 & & 1.04 & \\
\hline & & 2010 & 2011 & 2010 & 2011 & 2010 & 2011 \\
\hline \multirow{6}{*}{$0.2-0.4$} & $\mathrm{~T} 1$ & $0.57 \mathrm{bA}$ & $0.37 \mathrm{bB}$ & $33.50 \mathrm{aA}$ & $5.87 \mathrm{~dB}$ & $1.20 \mathrm{bA}$ & $1.50 \mathrm{bA}$ \\
\hline & $\mathrm{T} 2$ & $0.62 \mathrm{bA}$ & $0.37 \mathrm{bB}$ & $10.25 \mathrm{bA}$ & $5.00 \mathrm{dA}$ & $1.12 \mathrm{bA}$ & $1.45 \mathrm{bA}$ \\
\hline & T3 & $2.40 \mathrm{aA}$ & $2.42 \mathrm{aA}$ & $25.75 \mathrm{aA}$ & $38.50 \mathrm{bA}$ & $1.52 \mathrm{aA}$ & $1.65 \mathrm{bA}$ \\
\hline & T4 & $0.85 \mathrm{bA}$ & $0.55 \mathrm{bB}$ & $5.75 \mathrm{bA}$ & $11.75 \mathrm{dA}$ & $1.40 \mathrm{aA}$ & $1.22 \mathrm{cA}$ \\
\hline & T5 & $0.62 \mathrm{bA}$ & $0.30 \mathrm{bB}$ & $27.50 \mathrm{aA}$ & $35.75 \mathrm{bA}$ & $1.22 \mathrm{bA}$ & $1.45 \mathrm{bA}$ \\
\hline & T6 & $0.87 \mathrm{bA}$ & $0.57 \mathrm{bB}$ & $13.25 \mathrm{bB}$ & $48.50 \mathrm{aA}$ & $1.17 \mathrm{bB}$ & $1.55 \mathrm{bA}$ \\
\hline \multirow{6}{*}{$0.4-0.6$} & $\mathrm{~T} 1$ & $0.37 \mathrm{bA}$ & $0.45 \mathrm{bB}$ & $4.32 \mathrm{bA}$ & $4.90 \mathrm{dA}$ & $0.80 \mathrm{bA}$ & $0.90 \mathrm{cA}$ \\
\hline & $\mathrm{T} 2$ & $0.50 \mathrm{bA}$ & $0.25 \mathrm{bB}$ & 8.27 bA & $3.75 \mathrm{dA}$ & $0.82 \mathrm{bA}$ & $0.95 \mathrm{cA}$ \\
\hline & $\mathrm{T} 3$ & $1.55 \mathrm{bA}$ & $0.47 \mathrm{bB}$ & $31.75 \mathrm{aA}$ & $28.55 \mathrm{bA}$ & $1.00 \mathrm{bA}$ & $1.00 \mathrm{cA}$ \\
\hline & T4 & $1.30 \mathrm{bA}$ & $0.35 \mathrm{bB}$ & $20.00 \mathrm{bA}$ & $5.25 \mathrm{~dB}$ & $0.80 \mathrm{bA}$ & $0.87 \mathrm{cA}$ \\
\hline & T5 & $0.50 \mathrm{bA}$ & $0.55 \mathrm{bB}$ & $23.50 \mathrm{aA}$ & $6.52 \mathrm{~dB}$ & $0.82 \mathrm{bA}$ & $1.00 \mathrm{cA}$ \\
\hline & T6 & $0.55 \mathrm{bA}$ & $0.42 \mathrm{bB}$ & $8.75 \mathrm{bA}$ & $10.50 \mathrm{dA}$ & $0.80 \mathrm{bA}$ & $0.95 \mathrm{cA}$ \\
\hline
\end{tabular}

depths would be expected if gypsum $\left(\mathrm{CaSO}_{4}\right)$ was added to the soil (Shainberg et al., 1989), instead of only lime $\left(\mathrm{CaCO}_{3}\right)$. According to Djuric et al. (2011), the main effect of soil acidity is $\mathrm{Al}^{3+}$ toxicity, with the $\mathrm{Al}^{3+}$ saturation $(\mathrm{m})$ index being the best measure to assess this component of soil acidity and indicates the percentage of the effective soil cation exchange capacity (t) that is occupied by $\mathrm{Al}^{3+}$. Differences were found $(p<0.05)$ between treatments regarding $\mathrm{Al}^{3+}$ saturation (Table 2 ) at $0-0.2$ and $0.2-0.4 \mathrm{~m}$ soil depth. Therefore, no significant differences were observed $(p>0.05)$ in the 0.4-0.6 $\mathrm{m}$ soil depth. The lowest values for $\mathrm{Al}^{3+}$ saturation $(\mathrm{m})$ in the year of 2010 were found in treatments $\mathrm{T} 3, \mathrm{~T} 4, \mathrm{~T} 5$, and $\mathrm{T} 6$ at $0-0.2 \mathrm{~m}$ soil depth, while in 2011, the $\mathrm{Al}^{3+}$ saturation $(\mathrm{m})$ was low for T3 and T6 treatments. At $0.2-0.4 \mathrm{~m}$ soil depth, the $\mathrm{T} 3$ treatment presented the lowest $\mathrm{Al}^{3+}$ saturation, representing $47.1 \%$ of the cation exchange capacity (CEC). The treatments did not affect $\mathrm{Al}^{3+}$ saturation at $0.4-0.6 \mathrm{~m}$ soil depth. Oliveira et al. (2010) highlighted that $\mathrm{Al}^{3+}$ saturation in relation to CEC is one of the main factors limiting the sugarcane production.

\section{Soil exchangeable cations}

In the 0-0.2 m soil depth of 2010 it was observed an increase in the amount of $\mathrm{Ca}^{2+}$ in treatments T3 (liming-harrowingplowing-harrowing), T4 (desiccation-liming-direct planting), T5 (desiccation-liming-subsoiling) and T6 (harrowing-limingplowing-harrowing) (Table 3). In 2011, T3 and T4 treatments presented the highest $\mathrm{Ca}^{2+}$ levels. The soil $\mathrm{Ca}^{2+}$ availability is correlated with the soil $\mathrm{Al}^{3+}$ levels and $\mathrm{m}(\%)$. In this experiment, the no-till treatment (T4) presented $\mathrm{Ca}^{2+}$ content equivalent to treatments with soil revolving at the 
superficial soil layer (0-0.2 m). A great increase of soil acidity (low $\mathrm{pH}$ ) and $\mathrm{Al}^{3+}$ levels are regularly observed at soil layers below $0.4 \mathrm{~m}$ due to no influence of lime effects at such depths (Okorkov and Okorkova, 2013).

Conyers et al. (2003) discussed the movement of lime in soil surface and subsurface in no-till systems and concluded that the physical movement of lime in soil is probably through channels formed by dead roots left intact due to the lack of soil revolving, results that were not observed in this study. Almeida et al. (2005) observed increasing exchangeable $\mathrm{Ca}^{2+}$ content in no-till planting system with crop rotation in comparison to conventional system (soil revolving), up to 0.3 $\mathrm{m}$ deep, in a Cambisol in Santa Catarina state, Brazil. This was not the case observed in this study, where no differences between conventional tillage and no-tillage treatments were observed for $\mathrm{Ca}^{2+}$ content.

Regarding $\mathrm{Mg}^{2+}$ content in the soil, it was observed that the treatment which improve soil $\mathrm{Mg}^{2+}$ content at $0-0.2 \mathrm{~m}$ soil depth in 2010 was only T6; and in 2011, T3 and T6 presented significant increments in soil $\mathrm{Mg}^{2+}$ (Table 3). At 0.2-0.4 m soil depth, only $\mathrm{T} 3$ soil treatment increase soil $\mathrm{Mg}^{2+}$ content in both years. This situation can be explained by the efficiency of the lime incorporation in both treatments, which also presented similar results for $\mathrm{pH}, \mathrm{H}^{+}+\mathrm{Al}^{+3}, \mathrm{Al}^{+3}$ and $\mathrm{m}(\%)$.

The amount of $\mathrm{Mg}^{2+}$ at $0-0.20 \mathrm{~m}$ soil depth only differ for T1 (desiccation-liming-plowing-harrowing) and T3 (limingharrowing-plowing-harrowing) treatments (Table 3). This increment can be explained by lime solubilization and its residual effect increasing the amount of soil $\mathrm{Mg}^{2+}$ in 2011 . Nutrient cycling by sugarcane roots also can contribute increasing the amount of this nutrient in the soil. Sugarcane plants can develop roots of more than five meters long. These roots exude organic compounds which enrich the rhizosphere, improving the solubilization of soil components and the availability of nutrients in the soil solution. When sugarcane roots decompose there are also the releasing of substances that assist in the synthesis of humus along soil profile (Brady and Weil, 2013), improving diverse soil aspects.

At $0-0.2 \mathrm{~m}$ soil depth in 2010 , the $\mathrm{K}^{+}$concentrations for $\mathrm{T} 1$, T2, T3, and T6 treatments were superior (Table 3). In 2011, the soil $\mathrm{K}^{+}$concentration was great for the same treatments and also for T5 (desiccation-liming-subsoiling). At 0.2-0.4 m soil depth, T4 treatment (desiccation-liming-direct planting) resulted in small soil $\mathrm{K}^{+}$increment in 2011 when compared to the other treatments. The treatments did not affect the soil $\mathrm{K}^{+}$concentration at $0.2-0.4 \mathrm{~m}$ soil depth in 2010 , and at 0.4-0.6 $\mathrm{m}$ in both years.

Soil preparation can change the availability of $\mathrm{K}^{+}$, by providing more favorable conditions for root development, increasing the efficiency of water and nutrient uptake. These results are in agreement with those found by Pavinato et al. (2009). These authors reported that tillage systems promote changes in $\mathrm{K}^{+}$availability in superficial soil layers (up to 0.25 $\mathrm{m})$, while low $\mathrm{K}^{+}$availability was observed in no-tillage systems.

The soil base saturation $(\mathrm{V})$ at 0-0.2 $\mathrm{m}$ soil depth was great for T6 treatment in 2010; in 2011, the greatest soil base saturation was observed for T3 and T6 soil treatments. At 0.2-0.4 m soil depth, only T3 soil treatment resulted in increased soil base saturation. The evaluation periods ( 2010 and 2011) had no influence on the levels of $\mathrm{Ca}^{2+}, \mathrm{K}^{+}$and $\mathrm{V}$, even with better lime incorporation due to soil revolving, what would increase $\mathrm{Ca}^{2+}$ and $\mathrm{Mg}^{2+}$ availability in soil. However, in the evaluation of soil chemical attributes in sugarcane area at 0-0.2 m soil depth, Cury et al. (2014), found greater $\mathrm{Ca}^{2+}, \mathrm{P}$ and $\mathrm{V}$ in the no-tillage system than in conventional soil system. These diverging results can be attributed to experimental conditions, since the soil in our study was less fertile than that used by Cury et al. (2014).

Among treatments or evaluation years, no differences were observed at 0.4-0.6 $\mathrm{m}$ soil depth for soil $\mathrm{Ca}^{2+}, \mathrm{Mg}^{2+}, \mathrm{K}^{+}$and $\mathrm{V}$ (Table 3). These results were expected, especially in soil treatments where lime was incorporated at deep soil layers, as in the case of $T 1, T 2, T 3$, and $T 6$ treatments. Since lime could not percolate soil bases deep in soil profile its effects can only reach such depths with its incorporation using mechanical implements. Regularly, lime is incorporated at 0.15 to $0.2 \mathrm{~m}$ from soil surface; the $\mathrm{Ca}^{2+}$ and $\mathrm{Mg}^{2+}$ bases applied as lime participate of the CEC of the soil layer where it is deposited and do not readily move to deep soil layers (Brady and Weil, 2013).

Treatments T3 (liming-harrowing-plowing-harrowing), T4 (desiccation-liming-direct planting) and T5 (desiccationliming-subsoiling) presented increased $P$ levels at 0-0.2 m soil depth in 2010 (Table 4). In 2011, T3, T4 and T6 (harrowing-liming-plowing-harrowing) presented the greatest soil P. At 0.2-0.4 m soil depth, only T3 treatment significantly increase soil $P$ in both years. There was a reduction in $\mathrm{P}$ content at $0-0.20 \mathrm{~m}$ soil depth from 2010 to 2011 in T4 and T5 treatments. This macronutrient was not influenced by soil treatments at 0.4-0.6 $\mathrm{m}$ soil depth.

Busato et al. (2005) observed in systems that intend the plant residue accumulation increased $P$ levels in the $0-0.2 \mathrm{~m}$ soil depth, and maintenance of $P$ level in the $0.2-0.4 \mathrm{~m}$ soil depth; however, when there was pre-harvest sugarcane burning, lower $P$ levels were observed in both soil layers. Canellas et al. (2003), also observed a significant increase in the $\mathrm{P}$ content in a Cambisol long managed without preharvest burning. In this study, there were $P$ reductions above 50\% from 0-0.2 to 0.2-0.4 m soil depth, for most treatments. The increased P levels observed for T3, T5 and T6 treatments are associated with efficient lime incorporation which increases soil pH quickly, also increasing the availability of label $P$ in soil. The homogenous incorporation of lime promotes better effects of the applied product, providing indirect desirable results as better root development, consequently better water, and nutrient absorption reflecting positively in crop productivity.

The reduction in soil $\mathrm{P}$ content during the evaluation periods can be explained by the demands of the sugarcane crop and by $\mathrm{P}$ fixation in soils mineral components. The results found in this study show that in the no-tillage system also $P$ fixation occurs. The amount of $P$ was not affected in the soils from $\mathrm{T} 1, \mathrm{~T} 2, \mathrm{~T} 3$ and T6 treatments in both years. This happens as a function of the lime incorporation through soil revolving and its great contact with the reactive forms of the soil minerals (clay oxides).

Note that $\mathrm{T} 4$ treatment, where lime was applied followed by no-tillage planting and without desiccation, resulted in intermediary levels of all variables analyzed. This shows that no-tillage system can be applied without negatively affecting soil chemical characteristics at the depths evaluated. Although the no-tillage system had not provided large quantities of available nutrients in the soil during the period evaluated, it is expected that in the long term, the beneficial 
effects of this low soil disturbances system can be greater, economically and environmentally.

The no-tillage soil system intends to favor crop residues accumulation on the soil surface, without incorporating it in soil profile; therefore, it is also expected that over time, and with crop residue accumulation and cycling, the soil chemical, physical, biological properties, and fertility will be improved, directly affecting soil productive potential. Some authors, like Trivelin et al. (1997) and Strong et al. (2012), highlighted that the maintenance of crop residue on sugarcane areas and the application of mill waste from sugarcane industry (vinhaça) can benefit soil dynamics by improving nutrient cycling, reducing the use of industrial fertilizers and consequently reducing production costs.

The organic matter accumulation in no-tillage system is a key point to maintaining soil productive capacity in tropical environments, since it improves biological activity and nutrient cycling, and serves as an important source of nutrients to crop development, such as $N$, sulfur (S) and P. Sugarcane no-tillage, or minimum soil cultivation system, ensures greater levels of organic matter than conventional tillage system, which results in greater income and sustainability (Arruda et al., 2015). Another great economic gain from the adoption of no-tillage systems is the reduction of tillage operations, thus, reducing machinery, workinghour and fuel expenses.

In the 0.4-0.6 $\mathrm{m}$ soil depth, there was no influence of the treatments in the P content in both years (Table 4). This is due to the application of lime and P fertilizer at the same dosage for all treatments, and also due to limited effects of lime at this soil depth. In addition, $\mathrm{P}$ is available to plants in the anionic form of primary and secondary orthophosphate $\left(\mathrm{H}_{2} \mathrm{PO}_{4}{ }^{-}\right.$and $\left.\mathrm{HPO}_{4}{ }^{2-}\right)$, and as a negative charge, ion $\mathrm{P}$ presents low mobility in soils, which are mostly positive charged and holds $\mathrm{P}$ by strongly binding to its anionic forms (Vilar and Vilar, 2013). Similar soil pH levels in the superficial soil layers are possibly due to the presence of lime which makes $P$ more plant available. The opposite occurs in the deepest soil profile where $\mathrm{P}$ is bond with soil colloids due to higher acidity (low pH) and to the lack of applications of $P$ in this soil profile, thus, generating this similarity between treatments.

The T6 treatment increase $\mathrm{S}$ levels at the $0-0.2 \mathrm{~m}$ soil depth in 2010 (Table 4). Similar results for the treatments T4, T5 and T6 were observed in 2011. At 0.2-0.4 m soil depth, in the first year, the largest $S$ levels were found in T1, T3, and T5 treatments. In greatest soil depth evaluated, and T5 treatments resulted in the greatest soil S level in 2010, and T3 in 2011. The S increment is probably associated with mineral sources presented in the clay fraction. The soil in this study is rich in iron and aluminum oxides and in kaolinite, which has the capacity to strongly adsorb the existing sulfate in the soil solution. Subsequently, the sulfate is released slowly, by anionic exchange, especially at low soil $\mathrm{pH}$ (Brady and Weil, 2013).

The increase in soil $\mathrm{S}$ in the $0-0.2 \mathrm{~m}$ soil depth observed in T4 treatment can be attributed to the maintenance of the soil physical structure since this treatment was performed under no-tillage. The low soil revolving reduces the oxidation of organic matter and nutrient mineralization, maintaining soil physical structure. In conventional tillage systems of soil management there is increased organic matter mineralization, which is the main soil source of $S$, and increased organic carbon oxidation, which alters the soil $\mathrm{C} / \mathrm{N}$ ratio (Brady and Weil, 2013).

The reduction in $\mathrm{S}$ content may also be explained by the amount of charges present in the soil. Great amounts of negative charges in soil (electronegative) increases the repulsion on $\mathrm{S}_{-} \mathrm{SO}_{4}{ }^{-2}$ ion, improving $\mathrm{S}$ mobility and leaching capacity in the soil profile. The $\mathrm{S}$ mobility in $\mathbf{2 0 1 0}$ was higher than in 2011, and this occurred due to factors such as rain, with the average rainfall in 2010 experimental period equal to $0 \mathrm{~mm}$ and to $41.1 \mathrm{~mm}$ in 2011 .

The organic matter (OM) content in 0-0.2 m soil depth was not influenced by the treatments in 2010 (Table 4). An increased OM content at 0.2-0.4 m soil depth in 2010 was observed for T3 and T4 treatments. At 0-0.2 and 0.2-0.4 m soil depth, the treatment T4 resulted in low OM in 2011. The treatments did not affect this characteristic at $0.4-0.6 \mathrm{~m}$ soil depth. The evaluation periods also did not influence the soil OM content, excepting T6 treatment that resulted in great soil OM at 0.2-0.4 $\mathrm{m}$ soil depth in 2011 .

Silva et al. (2014) reported that no lime application along with no-tillage significantly reduced the loss of soil organic matter, however, the soil preparation with lime application resulted in $\mathrm{CO}_{2}$ loss $48 \%$ superior when compared to where there was no lime application. These results were not observed in this study. The results observed by Freitas et al. (2017) indicated that chemical attributes and organic matter are strongly influenced by soil use and management, similarly to what was found in this study.

\section{Materials and methods}

Two studies were conducted in 2010 and 2011 at Jalles Machado Sugarcane Mill located at the coordinates 15010' South and 49015' West, approximately 640 meters above sea level. The area was previously conducted as extensive pasture with Brachiaria decumbens without any soil chemical corrections over the past 10 years. The climate of the region is savannah tropical (Aw), with dry winters and rainy summers (Köppen, 1936). Changes in temperature and precipitation which occurred during the experiment are expressed in Figure 1.

The experiment was installed during January 2009. Tillage activities and the establishment of the soil management systems, which characterized the treatments, were done during the rainy period with the soil humidity close to field capacity. The soil is classified as Red-Yellow Dystrophic Latosol (EMBRAPA, 2018). Soil samples were collected from 0-0.2 and 0.2-0.4 m depths, to characterize soil initial conditions; analyses were made at the Jalles Machado Sugarcane Mill laboratory - texture analysis are in Table 1, initial chemical analyses are in Tables 2, 3 and 4.

The experiment was conducted with six treatments designed as complete randomized blocks due to field variable conditions, with four repetitions and evaluated in distinct years (2010 and 2011). Each parcel was $50 \mathrm{~m}$ long and 19.5 $\mathrm{m}$ wide - 13 sugarcane lines spaced $1.5 \mathrm{~m}$. Parcells and blocks were separated by $5 \mathrm{~m}$ wide alleys to enable machinery and implements maneuvers. The total experimental area was $34,505 \mathrm{~m}^{2}$.

Soil treatments were defined seeking sustainability and economic viability of farming systems on sugarcane expansion areas in the Cerrado biome (Brazilian Savanna like biome); the treatments were: 'desiccation-liming-plowing- 
harrowing' (T1); 'liming-plowing-harrowing' (T2); 'limingharrowing-plowing-harrowing' (T3); 'desiccation-limingdirect planting' (T4); 'desiccation-liming-subsoiling' (T5), and 'harrowing-liming-plowing-harrowing' (T6).

The plots of treatments including desiccation (T1, T4, and T5) were desiccated with glyphosate $\left(5 \mathrm{~L} \mathrm{ha}^{-1}\right) 30$ days before the soil treatments installation. Weed management during sugarcane cultivation included 1.4, 396 and $2.88 \mathrm{~g}^{-}{ }^{-}$ ${ }^{1}$ of diuron, hexazinone, and MSMA active ingredients. Spraying (JACTO ${ }^{\circledR}$ Condor $600 \mathrm{~L}, 12 \mathrm{~m}$ bar width and) was regulated to $75 \mathrm{~L} \mathrm{~min}^{-1}$ spray volume $\left(300 \mathrm{~L} \mathrm{ha}^{-1}\right)$, pressure of 50 psi, nozzle spray ( $110^{\circ}$ flat fan spray tip) every $0.5 \mathrm{~m}$ and located at 0.5 to $1.3 \mathrm{~m}$ above crop canopy.

The equivalent to $3.5 \mathrm{t} \mathrm{ha}^{-1}$ of dolomitic lime (relative power of total neutralization: 85\%) was uniformly spread (TATU ${ }^{\circledR}$ DCA MAXX 12000, weight 3,500 $\mathrm{kg}, 6 \mathrm{~m}^{3}$ capacity, lime spreading width of $16 \mathrm{~m}$ and $110 \mathrm{hp}$ work power) four months before planting in all treatments.

Plowing was done using a moldboard plow (TATU ${ }^{\circledR}$ AAH2, weight $560 \mathrm{~kg}$, with four moldboards spaced $0.6 \mathrm{~m}$ and work range of $2.4 \mathrm{~m}$ ) reaching $0.35-0.4 \mathrm{~m}$ soil depth at $5 \mathrm{~km} \mathrm{~h}^{-1}$ speed; harrowing with a leveling intermediate harrow (BALDAN ${ }^{\circledR}$ CRSG-L, weight $2,080 \mathrm{~kg}$, with 28 disks of $0.66 \mathrm{~m}$, spaced $0.235 \mathrm{~m}$, in two lines and work range of $3.2 \mathrm{~m}$ ) reaching $0.20-0.25 \mathrm{~m}$ soil depth at $5 \mathrm{~km} \mathrm{~h}^{-1}$ speed; subsoiling with a subsoiler $\left(\right.$ TATU $^{\circledR}$ Astimatic 500 , weight $2,200 \mathrm{~kg}$, with five shanks, spaced $0.4 \mathrm{~m}$, work range of $2 \mathrm{~m}$ and $160 \mathrm{hp}$ work power) reaching $0.4 \mathrm{~m}$ soil depth, and direct planting with a furrow reaching 0.3-0.4 $\mathrm{m}$ soil depth. When plowing, harrowing or subsoiling soil activities were part of the treatment they were applied only once.

Planting was done manually in April 2009 by placing 15-20 sugarcane buds $\mathrm{m}^{-2}$ at approximately $0.35 \mathrm{~m}$ soil depth. CTC2 sugarcane variety was used. During sugarcane planting, fertilizer was placed in the furrows at a dose of $250 \mathrm{~kg} \mathrm{ha}^{-1}$ of mono-ammonium phosphate, equivalent to $120 \mathrm{~kg} \mathrm{ha}^{-1} \mathrm{P}_{2} \mathrm{O}_{5}$ and $27 \mathrm{~kg} \mathrm{ha}^{-1}$ of $\mathrm{N}$. The furrows were soon covered after sugarcane bud distribution. Top dressing fertilization was carried out 130 days after planting with a liquid formula of 5$0-13+0.3 \% \mathrm{Zn}+0.3 \% \mathrm{~B}$, at the dose of 1,000 $\mathrm{Lha}^{-1}$.

After harvest, in July 2010 and July 2011, soil samples were taken from three different soil depths: 0-0.2, 0.2-0.4 and 0.4$0.6 \mathrm{~m}$. Three simple samples were randomly collected between the planting lines in each plot and then mixed to form a composite sample. The soil samples were sent to Federal University of Uberlândia (UFU), where soil $\mathrm{K}^{+}, \mathrm{S}^{-\mathrm{SO}_{4}}$, $\mathrm{Ca}^{2+}, \mathrm{Mg}^{2+}$ and $\mathrm{H}_{2} \mathrm{PO}_{4}^{-}$by Mehlich method $1 ; \mathrm{pH}$ in $\mathrm{H}_{2} \mathrm{O}$ (2.5:1); organic matter (OM) by colorimetric method; exchangeable acidity $\left(\mathrm{Al}^{3+}\right)$; total acidity $\left(\mathrm{H}^{+}+\mathrm{Al}^{3+}\right)$; aluminum saturation $(\mathrm{m}, \%)$, and base saturation $(\mathrm{V}, \%)$, where estimated according to methods described by EMBRAPA (2017).

The results were submitted to the basic assumptions of the analysis of variance (ANOVA) model and data transformation was required (square root of $x+0.5$ ). Appropriate ANOVA was done using $F$ test $(p<0.05)$, and subsequent, joint analysis between years and treatment mean comparisons were done using $t$-test $(p<0.05)$ and Tukey's test $(p<0.05)$, respectively. Statistical analyses were done using SISVAR (Ferreira, 2011) and GENES (Cruz, 2013) software.

\section{Conclusions}

In sugarcane expansion area soil class of fertility interferes in the chemical attributes and soil availability of nutrients to plants.

Farming practices such lime application followed by plowing and harrowing, greatly improve soil fertility in the initial years of sugarcane crop in expansion area.

The use of the no-tillage system (minimum cultivation system) is sustainable and viable in sugarcane cultivation, with the benefits of this system enhanced with time.

\section{Acknowledgments}

To FAPEMIG and CAPES for the student grants; to Jalles Machado Sugarcane Mill for all their support and research team.

\section{References}

Almeida JM, Bertol I, Leite D, Amaral AJ, Zoldan Jr WA (2005) Propriedades químicas de um cambissolo húmico sob o preparo convencional e semeadura direta após seis anos de cultivo. R Bras Ci Solo. 29(3): 437-445.

Alvarez V VH, Novais RF de, Barros NF de, Cantarutti RB, Lopes AS (1999) Interpretação dos resultados das análises de solos. In: Ribeiro AC, Guimarães PTG, Alvarez V VH (eds) Comissão de fertilidade do solo do Estado de Minas Gerais. Recomendações para o uso de corretivos e fertilizantes em Minas Gerais - 5 Aproximação, 20 th edn. UFV, Viçosa. 5.

Arruda EM, Almeida RF de, Silva Junior AC da, Ribeiro BT, Silva A de A, Lana RMQ (2015) Aggregation and organic matter in different tillage systems for sugarcane. Afr J Agric Res. 10(4): 281-288.

Benedini MS, Conde AJ (2008) Sistematização de área para a colheita mecanizada da cana-de-açúcar. R Coplana. Novembro, 23-25.

Brady NC, Weil RR (2013) Elementos da natureza e propriedades dos solos. 3 ed. Porto Alegre, Bookman. $686 p$.

Busato JG, Canellas LP, Velloso ACX (2005) Fósforo num Cambissolo cultivado com cana-de-açúcar por longo tempo: I - fracionamento seqüencial. R Bras Ci Solo. 29(6): 935-944.

Canellas LP, Velloso ACX, Marciano CR, Ramalho JFGP, Rumjanek VM, Rezende CE, Santos GA (2003) Propriedades químicas de um Cambissolo cultivado com cana-de-açúcar, com preservação do palhiço e adição de vinhaça por longo tempo. R Bras Ci Solo. 27(5): 935-944.

CONAB - Companhia Nacional de Abastecimento, 2017. Accessed in: 02/may/2017. Available in: <http://www.conab.gov.br/OlalaCMS/uploads/arquivos/1 7_04_20_14_04_31_boletim_cana_portugues_-_10_lev_17-18.pdf>.

Conyers MK, Heenan DP, Mcghie WJ, Poile GP (2003) Amelioration of acidity with time by limestone under contrasting tillage. Soil Tillage Res. 72(1): 85-94.

Cruz CD (2013) GENES: a software package for analysis in experimental statistics and quantitative genetics. Acta Scientiarum Agronomy. 35(3):271-276.

Cury TN, De Maria IC, Bolonhezi D (2014) Biomassa radicular da cultura de cana-de-açúcar em sistema convencional e 
plantio direto com e sem calcário. R Bras Ci Solo. 38(6): 1929-1938.

Djuric M, Mladenovic J, Pavlovic R, Murtic S, Milic V, Sekularac $G$ (2011) Aluminium content in leaf and root of oat (Avena sativa L.) grown on pseudogley soil. Afr J Biotechnol. 10(77): 17837-17840.

Duiker SW, Beegle DB (2006) Soil fertility distributions in long-term no-till, chisel/disk and moldboard plow/disk systems. Soil Tillage Res. 88: 30-41.

EMBRAPA - Empresa Brasileira de Pesquisa Agropecuária, (2017) Manual de métodos de análise de solo, 3 ed. EMBRAPA, Brasília.

EMBRAPA - Empresa Brasileira de Pesquisa Agropecuária. (2018) Sistema Brasileiro de Classificação de Solos. 5 ed. EMBRAPA Solos, Brasília.

Ferreira DF (2011) Sisvar: a computer statistical analysis system. Ciênc e Agrotec. 35(6): 1039-1042.

Foltran R (2008) Aplicação de calcário, silicato e gesso em soqueira de cana-de-açúcar sem despalha a fogo. $111 \mathrm{p}$. Dissertação de mestrado. Faculdade de Ciências Agronômicas da UNESP - Campus de Botucatu, SP.

Freitas L, Oliveira IA, Silva LS, Frare JCV, Filla VA, Gomes RP (2017) Indicadores da qualidade química e física do solo sob diferentes sistemas de manejo. Revista Unimar Ciências. 26(1-2): 1-18.

Inácio JB, Santos RJ (2014) A expansão canavieira no município de Delta-MG: "ilhados pelos canaviais". Caminhos de Geografia. 14(46): 209-227.

Kaminski J, Silva LS, Ceretta CA, Rheinheimer D (2007) Acidez e calagem em solos do sul do Brasil: aspectos históricos e perspectivas futuras. In: Ceretta CA, Silva LS, Reichert JM (Eds.) Tópicos em ciência do solo, v. 1. UFV, Viçosa. 6.

Köppen W (1936) Das geographische system der klimate. In: Köppen W, Geiger R (Eds) Handbuch der Klimatologie. Gebrüder Borntraeger, Berlin. pp. 1-44.

Novais RF, Alvarez V VH, Barros NF, Fontes RL, Cantarutti RB, Neves JCL (2007) Fertilidade do Solo. SBCS, Viçosa. 1017p.

Okorkov VV, Okorkova LA (2013) Interaction of lime and gypsum with sorption complex of acidic soils. Russ Agric Sci. 39(5): 459-463.
Oliveira CMR, Passos RR, Andrade FV, Reis EF, Sturm GM, Souza RB de (2010) Corretivos da acidez do solo e níveis de umidade no desenvolvimento da cana-de-açúcar. Rev Bras. Ciênc Agr. 5(1): 25-31.

Pavinato PS, Merlin A, Rosolem CA (2009) Disponibilidade de cátions no solo alterada pelo sistema de manejo. Rev Bras Ciênc Solo. 33(4): 1031-1040.

Raij BV (2011) Fertilidade do solo e manejo de nutrientes. International Plant Nutrition Institute, Piracicaba. $420 \mathrm{p}$.

Rossiello ROP, Jacob Neto J (2006) Toxidez de alumínio em plantas: novos enfoques para um velho problema. In: Fernades MS (ed.) Nutrição mineral de plantas. SBCS, Viçosa. 15.

Shainberg I, Sumner ME, Miller WP, Farina MPW, Pavan MA, Fey MV (1989) Use of gypsum on soils: A review. Adv Soil Sci. 9: 1-101.

Silva E de F, Moitinho MR, Teixeira D de B, Pereira GT, Scala Junior N La (2014) Emissão de $\mathrm{CO}_{2}$ do solo associada à calagem em área de conversão de laranja para cana-deaçúcar. Enge Agríc. 34(5): 885-898.

Strong DJ, Flecker R, Valdes PJ, Wilkinson IP, Rees JG, Zong YQ, Lloyd JM, Garrett E, Pancost RD (2012) Organic matter distribution in the modern sediments of the Pearl River Estuary. Org Geochem. 49: 68-82.

Trivelin PCO, Berdassolli JA, Oliveira MW (1997) Potencialidade da mistura de aquamônia com vinhaça na fertilização de canaviais colhidos sem despalha a fogo: Estabilidade química da mistura. STAB - Açúcar. Álcool Subpr. 16(2): 26-29.

Vilar CC, Vilar FCM (2013) Comportamento do fósforo em solo e planta. Ci Exatas Terra Ci Agr. 8(2): 37-44.

Vitti GC, Mazza JA (2002) Planejamento, estratégias de manejo e nutrição da cultura de cana-de-açúcar. Potafos Informações Agronômicas, Piracicaba. 97.

Weirich Neto, PH, Caires EF, Justino A, Dias J (2000) Correção da acidez do solo em função de modos de incorporação de calcário. Ciênc Rural. 30 (2): 257-261. 